\title{
Simulation Study of BSC Racing Modeling and Handling Stability Based on CarSim
}

\author{
Shuhua Liao ${ }^{1, ~ a}$, Kaibin Cao, b, Xu Zhang ${ }^{1}$, Jiong Li ${ }^{1}$, Jingsheng Yang ${ }^{1}$ \\ ${ }^{1}$ College of Automobile and Transportation, Guangxi University of Science and Technology, China \\ aliaohsh60@163.com, b501333069@qq.com
}

Keywords: BSC Racing, Handling Stability, Modeling, Simulation, CarSim

\begin{abstract}
Pointing to the characteristics of the tournament of Baja SAE China, this paper analyzes the handling and stability of the whole vehicle during the project development of Baja Racing. A whole vehicle dynamics model based on CarSim software is built. Using 3D software to draw the three-dimensional vehicle model and importing it into CarSim to realize vehicle animation simulation. According to the requirements of the competition to set up the steady state cornering test conditions and the fish hook test conditions in the CarSim for analyzing and evaluating the handling and stability of the Baja Racing. The simulation result illustrated that the developed Baja Racing has good handling and stability.
\end{abstract}

\section{Introduction}

The Baja SAE China [1] is a competition for the design, manufacture and testing of off-road vehicles organized by students from colleges, universities and vocational schools. In accordance with the rules of the race and the car manufacturing standard, the Racing Team use the same type of engine at the specified time to design and manufacture a single seat, middle-engine and rear-drive small off-road vehicle. The tournament adopts the way of combination of static and dynamic games. The static items include technical inspection and design responses. The dynamic items include climbing tests, straight-line testing, handling testing and endurance testing. Among them, the test and evaluation of the handling and stability of the car accounted for $25 \%$ of the total score.

This paper analyzes the handling and stability of the whole vehicle during the project development of Baja Racing of the Guangxi University of Science and Technology. Adopt CarSim software to build a whole vehicle dynamics model. In UG Software, the three-dimensional vehicle model is drawn and imported into CarSim, so that it can visually simulate the motion of the Baja Racing under various operating conditions. Simulating and analyzing the handling and stability of the Baja Racing by setting the steady state cornering test conditions and the fish hook test conditions [2].

\section{Building the Baja Racing model based on CarSim}

CarSim software is a multi-body dynamics software based on system characteristic parameter modeling. It combines the traditional vehicle dynamics and the modern multi-body dynamics modeling method to abstract and simplify the vehicle. In contrast to Adams software is widely used in the Baja Racing dynamics modeling, this modeling method does not need to consider the specific structure of the system, hard point coordinates, bushing characteristics and the mass of the link, and other detailed parameters, but will reflect the system performance of the inertial parameters, stiffness parameters, damping parameters and other information input can be completed to build the whole vehicle dynamics model [3]. CarSim software mainly consists of three parts, model input, analysis process and result output. Its professional performance is good, especially suitable for the whole vehicle development process in the early stage of performance analysis and it can be more accurate simulation of the actual vehicle stability test.

Design and calculate the basic structure parameters and characteristic parameters of each 
subsystem of the Baja Racing [4, 5], and input these parameters into the Carsim software. Set up each subsystem model, including vehicle body, steering system, suspension system, tire, braking system and drive system, thus build a whole vehicle model. In the whole vehicle development process, part parameters of the Baja Racing as shown in Tab.1.

Tab.1 Part parameters of the whole vehicle model

\begin{tabular}{|c|c|c|c|}
\hline Parameter & Value & Parameter & Value \\
\hline Height /mm & 1470 & Sprung Mass/kg & 182 \\
\hline Width/mm & 1560 & Front Wheel Load /kg & 114 \\
\hline Wheel Base/mm & 1450 & Rear Wheel Load /kg & 20 \\
\hline Front Wheel Track/mm & 1380 & Front Suspension Stiffness/ N*mm & -1 \\
\hline Rear Wheel Track /mm & 1320 & Rear Suspension Stiffness / N*mm & 25 \\
\hline Height of Mass Center $/ \mathrm{mm}$ & 302 & Effective Wheel Rolling Radius/mm & 278 \\
\hline
\end{tabular}

\subsection{Vehicle body parameters}

The vehicle body model is established as shown in Fig.1. Input basic parameters, the body height is $1470 \mathrm{~mm}$, width is $1560 \mathrm{~mm}$, the whole vehicle sprung mass is $182 \mathrm{~kg}$, wheelbase is $1450 \mathrm{~mm}$, height of mass center is $302 \mathrm{~mm}$ and the distance of mass center from the front axle is $735 \mathrm{~mm}$ etc.

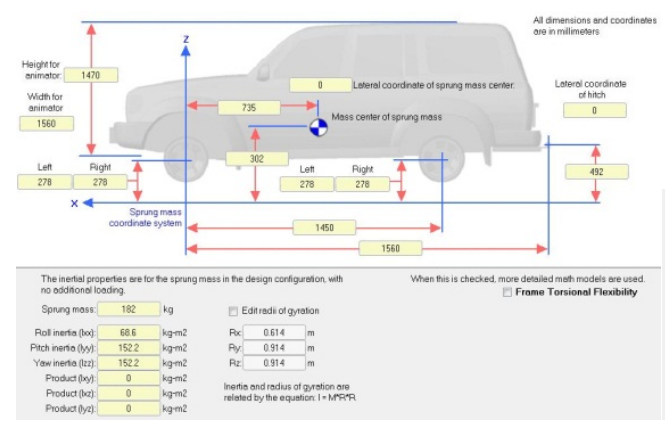

Fig.1 Vehicle body parameters
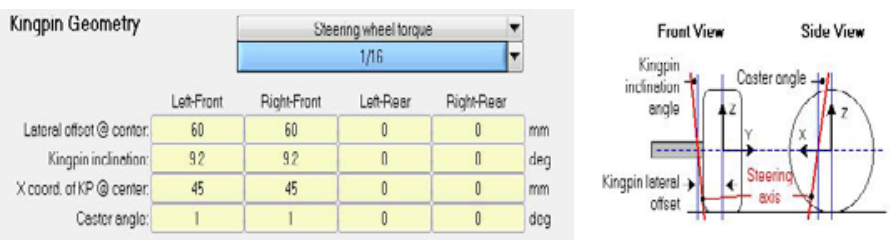

Fig.2 Steering System

\subsection{Steering System}

The steering system model is built as shown in Fig.2. Input basic parameters, the kingpin inclination angle is $9.2 \mathrm{deg}$, caster angle is $1 \mathrm{deg}$, the lateral offsets is $60 \mathrm{~mm}$, longitudinal offsets is $45 \mathrm{~mm}$ and the force transmission ratio of steering system is $1 / 16$ etc.

\subsection{Suspension System}

The suspension system model is established as shown in Fig.3 and Fig.4.

Input basic parameters of the front suspension system, unsprung mass is $30 \mathrm{~kg}$, camber angle is $0.5 \mathrm{deg}$, toe angle is $1 \mathrm{deg}$, sprung stiffness is $20 \mathrm{~N} / \mathrm{mm}$ and the damping is $2.4 \mathrm{~N} /(\mathrm{mm} / \mathrm{s})$ etc.

Input basic parameters of the rear suspension system, unsprung mass is $33 \mathrm{~kg}$, sprung stiffness is $25 \mathrm{~N} / \mathrm{mm}$ and the damping is $3.6 \mathrm{~N} /(\mathrm{mm} / \mathrm{s})$ etc.

\subsection{Tire model}

Considering that the Baja Racing Tire model is close to the car tire model in CarSim, the tire model chooses the Racing Tire model in the CarSim software. As the front and rear wheel assemblies are identical, input basic parameters of the wheel model, effective wheel rolling radius is $278 \mathrm{~mm}$, free radius is $280 \mathrm{~mm}$ and the stiffness is $220 \mathrm{~N} / \mathrm{mm}$, etc., as shown in Fig.5.

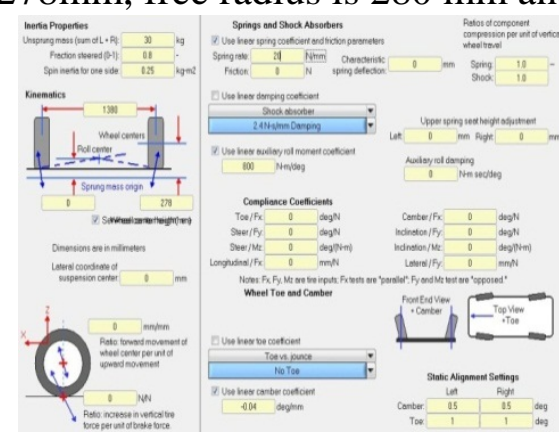

Fig.3 Front Suspension System

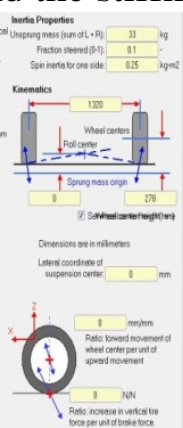

Fig.4 Rear Suspension System

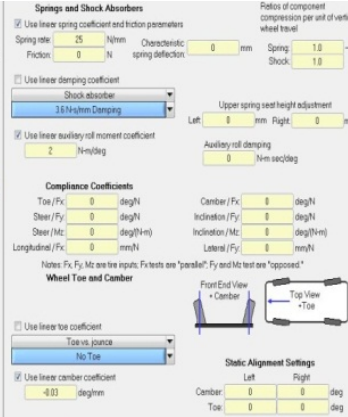

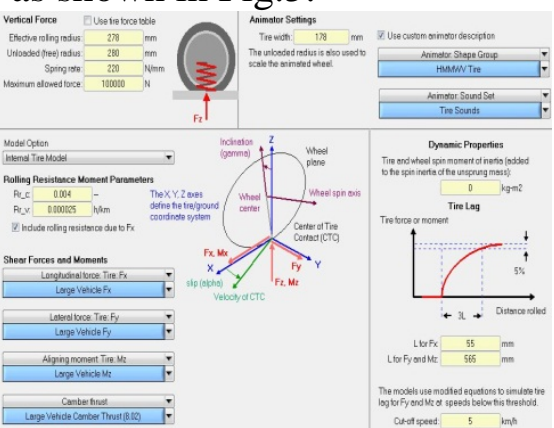

Fig.5 Tire model 


\subsection{Drive System and Braking System}

Taking into account that the handling and stability test is a constant speed test, on the basis of the adjustment ratio of 12.28 and the power distribution of $7: 3$, the default values of the racing drive system and braking system in CarSim are selected, as shown in Fig.6 and Fig.7.

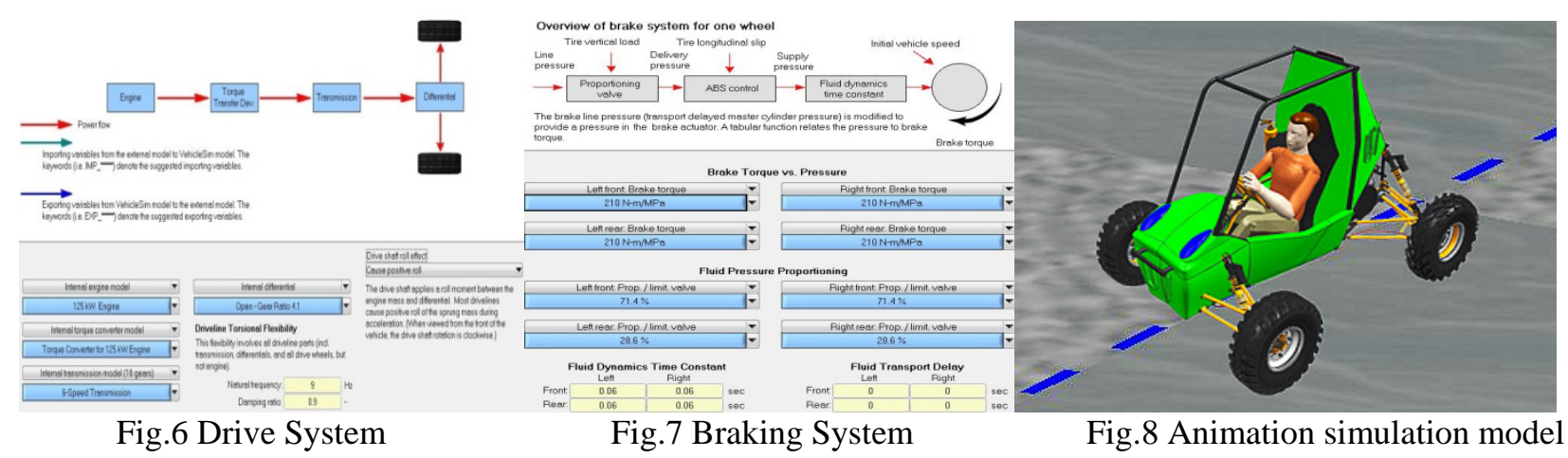

\subsection{Animation simulation model}

In order to be able to visually reflect the movement of the developed Baja Racing in the simulation animation, 3D software was used to design the three-dimensional model of frame, body, seat, suspension, wheel and tire, and imported it into CarSim software to get the three-dimensional solid model of animation simulation, as shown in Fig.8.

\section{Simulation and analysis of the handling stability of the Baja Racing}

According to the BSC Racing Rules and GB/T 6323-2014 Vehicle Handling and Stability Test Method, the steady-state cornering test conditions and the fish hook test conditions are selected to simulate the established Baja Racing model.

\subsection{Steady-state cornering test}

\subsubsection{Test method}

There are two kinds of the steady-state cornering test methods, Constant Steering Wheel Cornering Method and Constant Radius Cornering Method.

During the test, the vehicle runs at the lowest steady speed, adjusts the steering wheel angle, causes the vehicle to carry on the circle movement along the radius $30 \mathrm{~m}$ path. When the vehicle enters the circular path and reaches the stable state, the driver begins to record and keep the speed and steering wheel corner position within 3s. Gradually increase the speed, so that the lateral acceleration of the vehicle does not increase more than $0.5 \mathrm{~m} / \mathrm{s}^{2}$, until the lateral acceleration reaches $6.5 \mathrm{~m} / \mathrm{s}^{2}$, the test is finished. The steady-state cornering test scenario in CarSim is shown in Fig.9.

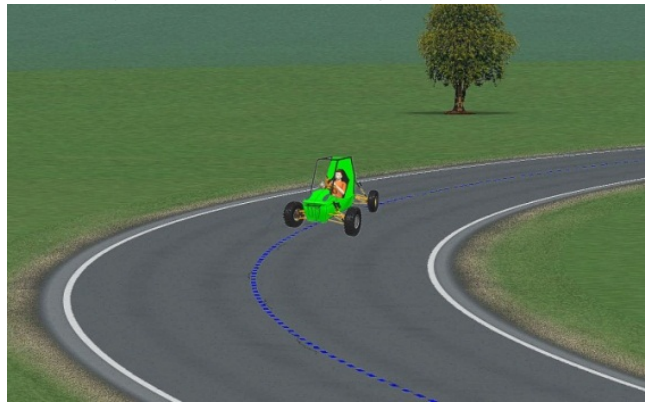

Fig.9 Steady-state cornering test scenario

\subsubsection{Analysis and evaluation of test results}

The difference of the slip angle between the front axle and rear axle is $\delta_{1}-\delta_{2}$, and is determined by the formula (1):

$$
\delta_{1}-\delta_{2}=57.3 \cdot \frac{L}{R} \cdot\left(\frac{\theta_{\mathrm{k}}}{\theta_{0}}-1\right)
$$

In the formula, $\delta_{1}$ and $\delta_{2}$ are respectively the front and rear axles slip angles. The $\theta_{0}$ is the steering wheel angle when the vehicle passes through the circular path at lowest steady speed, and the $\theta_{\mathrm{k}}$ is the steering wheel angle when the car passes the circular path at a certain speed. $\mathrm{L}$ is the 
wheelbase, and $\mathrm{R}$ is the radius of the circular path.

The simulation results of the steady-state cornering test with constant radius cornering method are shown in Fig.10 and Fig.11.

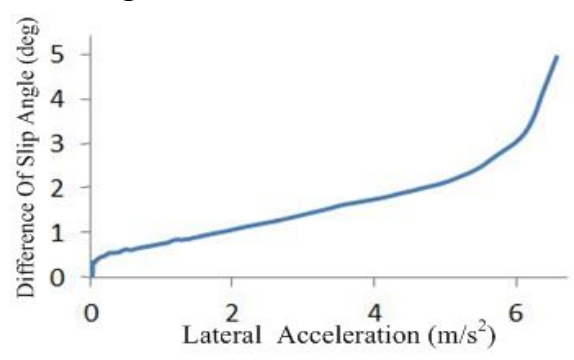

Fig.10 Difference of Slip Angle with Lateral Acc. Curve

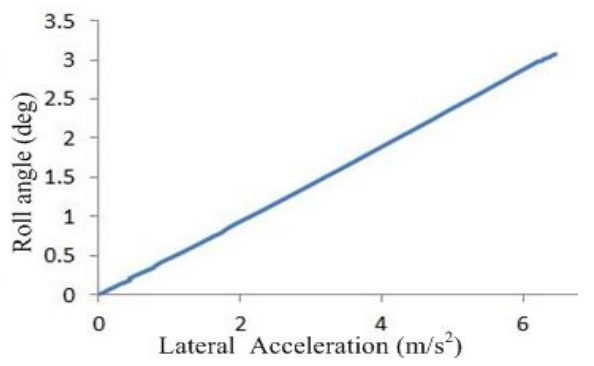

Fig.11 Roll Angle with Lateral Acc. Curve

According to the QC/T 480-1999 Vehicle Handling and Stability Index Limit and Evaluation Method [6], the steady-state cornering characteristics are evaluated and scored.

1) The evaluation score of the lateral acceleration value of a Neutral turning point is defined as $\mathrm{Na}_{\mathrm{n}}$. When no neutral point occurs, the score is recorded as 100 .

$$
N a_{\mathrm{n}}=60+\frac{40}{a_{\mathrm{n} 100}-a_{\mathrm{n} 60}} \cdot\left(a_{n}-a_{n 60}\right)
$$

In the formula, the $a_{\mathrm{n}}$ is the lateral acceleration value of the slope of zero at the relation curve between the difference of the slip angle between the front axle and rear axles and the lateral acceleration. The $a_{\mathrm{n} 100}$ and $a_{\mathrm{n} 60}$ are respectively the upper and lower values of the lateral acceleration of the neutral turning point.

2) The evaluation score value of Under-steer degree is defined as $\mathrm{N}_{U}$.

$$
\begin{gathered}
\lambda=60+\frac{2 \cdot U_{60} / U_{100}}{U_{60} / U_{100}-2} \cdot U_{100} \\
N_{\mathrm{U}}=60+\frac{U\left(U_{60}-U\right)(\lambda-U)}{U_{100}\left(U_{60}-U_{100}\right)\left(\lambda-U_{100}\right)} \cdot 40
\end{gathered}
$$

In the formula, $\lambda$ is the ratio coefficient, $U$ is the test value of the under-steer degree, and is the slope value of the lateral acceleration at the $2 \mathrm{~m} / \mathrm{s}^{2}$ point between the front and rear axles slip angles difference and the lateral acceleration curve. The $\mathrm{U}_{100}$ and $\mathrm{U}_{60}$ are respectively the upper and lower values of the Under-steer degree.

3) The evaluation score value of Carriage reclining degree is defined as $\mathrm{N}_{\phi}$.

$$
N_{\phi}=60+\frac{40}{K_{\phi 60}-K_{\phi 100}} \cdot\left(K_{\phi 60}-K_{\phi}\right)
$$

In the formula, $K_{\phi}$ is the test value of the Carriage reclining degree, and is the slope value of the lateral acceleration at the $2 \mathrm{~m} / \mathrm{s}^{2}$ point between the roll angle and the lateral acceleration curve. The $K_{\phi 100}$ and $K_{\phi 60}$ are respectively the upper and lower values of the Carriage reclining degree.

The comprehensive evaluation score of the steady-state cornering simulation test is defined as $N_{w}$ and calculated according to formula (6).

$$
N_{w}=\frac{N a_{n}+N_{U}+N_{\phi}}{3}
$$

The evaluation score values in each state are shown in Tab.2.

Tab.2 The evaluation scoring value of steady-state cornering simulation test

\begin{tabular}{|c|c|c|c|c|}
\hline Parameter & $N a_{n}$ & $N_{U}$ & $N_{\phi}$ & $N_{w}$ \\
\hline Value & 100 & 86 & 95.8 & 93.93 \\
\hline
\end{tabular}

From the above table, the evaluation score value of Under-steer degree is low. But overall, the steady-state cornering simulation test to carry out a comprehensive evaluation of the score value $N_{w}$ $=93.93$, it shows that the vehicle has good performance under the steady-state cornering test of the constant radius cornering.

\subsection{Fish hook test}

\subsubsection{Test method}

According to the test method of Fish hook condition in the literature, 10 piles are arranged in the CarSim virtual test field as shown in Fig.12, of which $L=30 \mathrm{~m}, \mathrm{~B}=2.5 \mathrm{~m}$. The Fish hook test scenario in CarSim shown in Fig.13, the Baja Racing passes through these standard piles at $65 \mathrm{~km} / \mathrm{h}$ speed. 


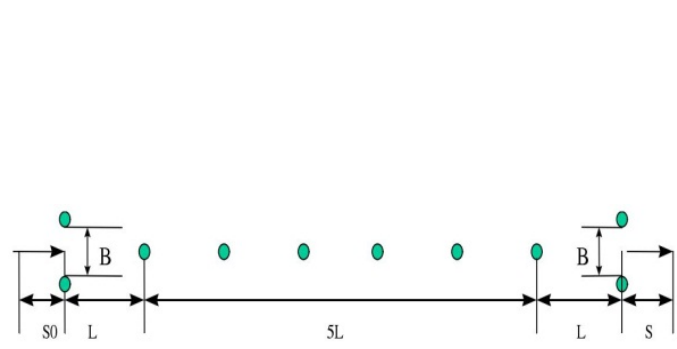

Fig.12 the standard pile diagram of the Fish hook test site

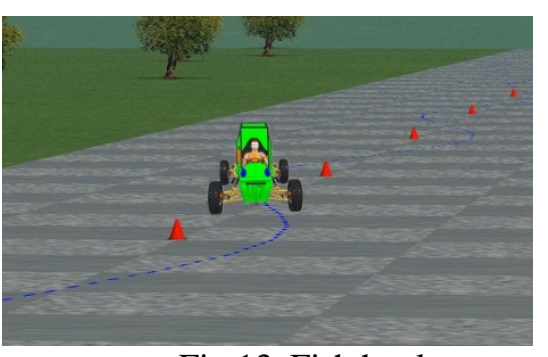

Fig.13 Fish hook test scenario

\subsubsection{Analysis and evaluation of test results}

The simulation results of the Fish hook test condition are shown in Fig.14-16.

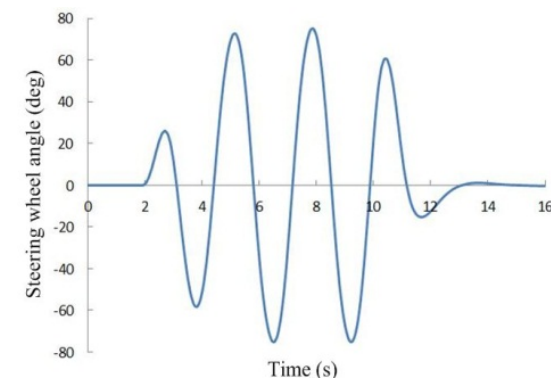

Fig.14 Steering Wheel Angle Curve

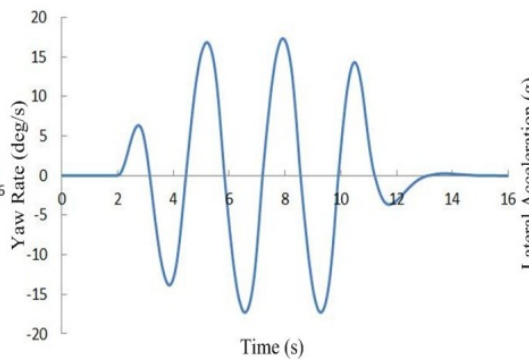

Fig.15 Yaw Rate Curve

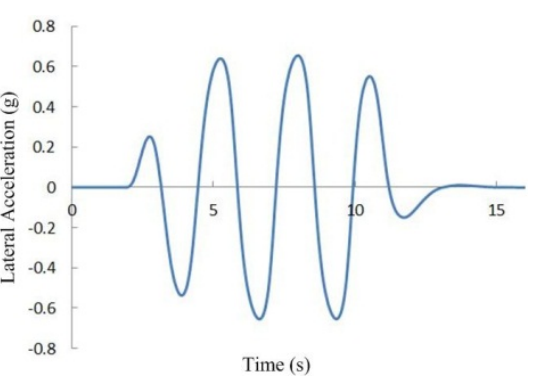

Fig.16 Lateral Acceleration Curve

According to the literature [6], the Fish hook test characteristics are evaluated and scored.

1) The evaluation score of the average peak value of steering wheel angle is defined as $N_{\theta}$.

$$
N_{\theta}=60+\frac{40}{\theta_{60}-\theta_{100}} \cdot\left(\theta_{60}-\theta\right)
$$

In the formula, $\theta$ is the test value of the average peak value of steering wheel angle at $65 \mathrm{~km} / \mathrm{h}$ speed. The $\theta_{100}$ and $\theta_{60}$ are respectively the upper and lower values of the average peak value of steering wheel angle.

2) The evaluation score of the average peak value of yaw rate is defined as $\mathrm{N}_{\mathrm{r}}$.

$$
N_{r}=60+\frac{40}{r_{60}-r_{100}} \cdot\left(r_{60}-r\right)
$$

In the formula, $r$ is the test value of the average peak value of yaw rate at $65 \mathrm{~km} / \mathrm{h}$ speed. The $\mathrm{r}_{100}$ and $r_{60}$ are respectively the upper and lower values of the average peak value of yaw rate.

The comprehensive evaluation score of the fish hook simulation test is defined as $N_{s}$ and calculated according to formula (9).

$$
N_{\mathrm{s}}=\frac{N_{\theta}+2 N_{r}}{3}
$$

The experimental data of the Fish Hook Test Performance Index are shown in tab.3.

Tab.3 The Fish Hook Test Performance Index

\begin{tabular}{|c|c|c|c|c|c|c|}
\hline Parameter & $\theta / \mathrm{deg}$ & $\mathrm{r} /\left(\mathrm{deg} \cdot \mathrm{s}^{-1}\right)$ & $\mathrm{a} /(\mathrm{g})$ & $N_{\theta}$ & $N_{r}$ & $N_{s}$ \\
\hline Value & 74.62 & 13.9 & 0.58 & 94.72 & 89.6 & 91.31 \\
\hline
\end{tabular}

By calculation, the comprehensive evaluation score of the fish hook test is $N_{s}=91.31$. This result is higher than the average 70 points of the automotive industry standard, which shows that the handling and stability of the whole vehicle model built by Fig. 1 to Fig.7 is very good under the fish hook conditions, and the vehicle responds stably and quickly.

\section{Conclusions}

According to the basic structure and characteristic parameters of the developed Baja Racing by our university, the whole vehicle model is built with the vehicle dynamics software CarSim. Selected the steady state cornering test conditions and the fish hook test conditions to analyze and evaluate the handling and stability of the Baja Racing. The simulation result illustrated that the developed Baja Racing has good handling and stability. 


\section{References}

[1] The rules of Baja SAE China [S].Beijing: SAE China, 2016.

[2] GB/T 6323-2014. Vehicle Handling and Stability Test Method.[S].

[3] Lijuan Jia. Simulation Study of FSC Racing Car Modeling and Handling Stability Based on CarSim [J]. Journal of Liaoning University of Technology, 2014.(3):172-176.

[4] Wangyu Wang. Automobile Design [M].Beijing: China Machine Press, 2002.

[5] Fan Yu. Automotive System Dynamics [M].Beijing: China Machine Press, 2012.

[6] QC-T 480-1999. Vehicle Handling and Stability Index Limit and Evaluation Method.[S]. 